\title{
Complete genome sequence of Sebaldella termitidis type strain (NCTC 11300')
}

\author{
Miranda Harmon-Smith ${ }^{1}$, Laura Celia ${ }^{2}$, Olga Chertkov ${ }^{3}$, Alla Lapidus ${ }^{1}$, Alex Copeland ${ }^{1}$, \\ Tijana Glavina Del Rio ${ }^{1}$, Matt Nolan ${ }^{1}$, Susan Lucas ${ }^{1}$, Hope Tice ${ }^{1}$, Jan-Fang Cheng ${ }^{1}$, Cliff \\ Han $^{1,3}$, John C. Detter ${ }^{1,3}$, David Bruce ${ }^{1,3}$, Lynne Goodwin ${ }^{1,3}$, Sam Pitluck ${ }^{1}$, Amrita Pati ${ }^{1}$, \\ Konstantinos Liolios ${ }^{1}$, Natalia Ivanova ${ }^{1}$, Konstantinos Mavromatis ${ }^{1}$, Natalia Mikhailova ${ }^{1}$, \\ Amy Chen ${ }^{4}$, Krishna Palaniappan ${ }^{4}$, Miriam Land ${ }^{1,5}$, Loren Hauser ${ }^{1,5}$, Yun-Juan Chang ${ }^{1,5}$, \\ Cynthia D. Jeffries ${ }^{1,5}$, Thomas Brettin ${ }^{1,3}$, Markus Göker ${ }^{6}$, Brian Beck ${ }^{2}$, James Bristow ${ }^{1}$, \\ Jonathan A. Eisen ${ }^{1,7}$, Victor Markowitz ${ }^{4}$, Philip Hugenholtz ${ }^{1}$, Nikos C. Kyrpides ${ }^{1}$, Hans-Peter \\ Klenk $^{6^{*}}$, and Feng Chen ${ }^{1}$ \\ ${ }^{1}$ DOE Joint Genome Institute, Walnut Creek, California, USA \\ ${ }^{2}$ ATCC- American Type Culture Collection, Manassas, Virginia, USA \\ ${ }^{3}$ Los Alamos National Laboratory, Bioscience Division, Los Alamos, New Mexico, USA \\ ${ }^{4}$ Biological Data Management and Technology Center, Lawrence Berkeley National \\ Laboratory, Berkeley, California, USA \\ ${ }^{5}$ Oak Ridge National Laboratory, Oak Ridge, Tennessee, USA \\ ${ }^{6}$ DSMZ - German Collection of Microorganisms and Cell Cultures GmbH, Braunschweig, \\ ${ }^{7}$ University of California Davis Genome Center, Davis, California, USA \\ ${ }^{*}$ Corresponding author: Hans-Peter Klenk
}

Keywords: anaerobic, mesophile, nonmotile, non-sporeforming, Gram-negative, termite intestine, 'Fusobacteria', 'Leptotrichiaceae', GEBA

Sebaldella termitidis (Sebald 1962) Collins and Shah 1986, is the only species in the genus Sebaldella within the fusobacterial family 'Leptotrichiaceae'. The sole and type strain of the species was first isolated about 50 years ago from intestinal content of Mediterranean termites. The species is of interest for its very isolated phylogenetic position within the phylum Fusobacteria in the tree of life, with no other species sharing more than $90 \% 16 \mathrm{~S}$ rRNA sequence similarity. The 4,486,650 bp long genome with its 4,210 protein-coding and 54 RNA genes is part of the Genomic Encyclopedia of Bacteria and Archaea project.

\section{Introduction}

Strain NCTC $11300^{\mathrm{T}}$ (= ATCC $33386^{\mathrm{TM}}=$ NCTC 11300 ) is the type strain of the species Sebaldella termitidis [1]. The strain was first isolated from posterior intestinal content of Reticulitermes lucifugus (Mediterranean termites) by the French microbiologist Madeleine Sebald [1,2], and was initially classified as Bacteroides termitidis [3]. The unusually low $\mathrm{G}+\mathrm{C}$ content, as well as biochemical features which did not correspond to those known for the other members of the genus Bacteroides [4], and the subsequently described novel $16 \mathrm{~S}$ rRNA sequences [5] made the position of B. termitidis within the genus Bacteroides appear controversial, and guided Collins and Shah in 1986 to reclassify $B$. termitidis as the type strain of the novel genus Sebaldella [1]. Here we present a summary classification and a set of features for $S$. termitidis NCTC $11300^{\mathrm{T}}$, together with the description of the complete genomic sequencing and annotation.

\section{Classification and features}

NCTC $11300^{\mathrm{T}}$ represents an isolated species, with no other cultivated strain known in the literature belonging to the species. An uncultured clone with identical 16S rRNA sequence was identified in a mesophilic anaerobic digester that treats municipal wastewater sludge in Clos de Hilde, France [6], and another uncultured clone, PCD-1 (96.1\% 16S rRNA sequence identity), was reported from the 
digestive tract of the ground beetle Poecilus chalcites [7]. The closest related type strains are those of the genus Leptotrichia, which share 85.9 to 89.96\% 16S rRNA sequence similarity [8]. Neither environmental screenings nor metagenomic surveys provided any $16 \mathrm{~S}$ rRNA sequence with significant sequence similarity to NCTC $11300^{\mathrm{T}}$, indicating that members of the species $S$. termitidis and the genus Sebaldella are not very frequent in the environment (status February 2010).
Figure 1 shows the phylogenetic neighborhood of $S$. termitidis NCTC $11300^{\mathrm{T}}$ in a $16 \mathrm{~S}$ rRNA based tree. The sequences of the four identical copies of the 16S rRNA gene in the genome do not differ from the previously published 16S rRNA sequence generated from ATCC 3386 (M58678), which is missing two nucleotides and contains 30 ambiguous base calls.

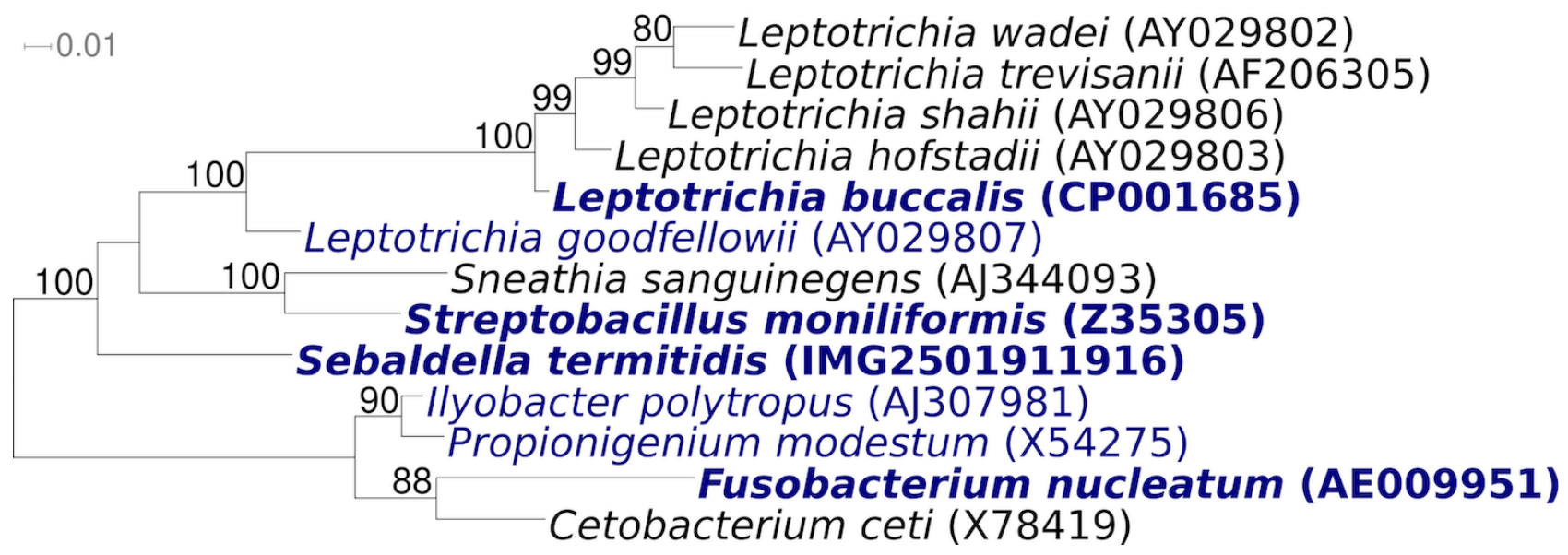

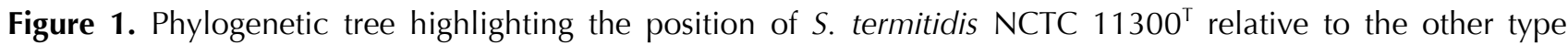
strains within the family 'Leptotrichiaceae'. The tree was inferred from 1,422 aligned characters $[9,10]$ of the $16 \mathrm{~S}$ rRNA gene sequence under the maximum likelihood criterion [11] and rooted in accordance with the current taxonomy. The branches are scaled in terms of the expected number of substitutions per site. Numbers above branches are support values from 1,000 bootstrap replicates if larger than $60 \%$. Lineages with type strain genome sequencing projects registered in GOLD [12] are shown in blue, published genomes in bold, e.g. the recently published GEBA genomes from Leptotrichia buccalis [13], and Streptobacillus moniliformis [14].

Cells of strain NCTC $11300^{\mathrm{T}}$ are Gram-negative, obligately anaerobic, nonmotile, nonsporeforming rods of 0.3 to $0.5 \times 2$ to $12 \mu \mathrm{m}$ with central swellings (Figure 2 and Table 1) [1]. Cells occur singly, in pairs, as well as in filaments [1]. Colonies on surface are transparent to opaque, circular measuring 1-2 $\mathrm{mm}$ in diameter, whereas colonies in deep agar are non pigmented and lenticular [1].

The major end products of the glucose metabolism by strain NCTC $11300^{\mathrm{T}}$ are acetic and lactic acids (with some formic acid) as opposed to succinic and acetic acids dominating in members of the genus Bacteroides [1]. Enzymes of the hexosemonophosphate-shunt are missing, while present in members of the genus Bacteroides [1,4]. A list of additional sugars and alcohols used or not-used for fermentation is provided by Collins and Shah [1].

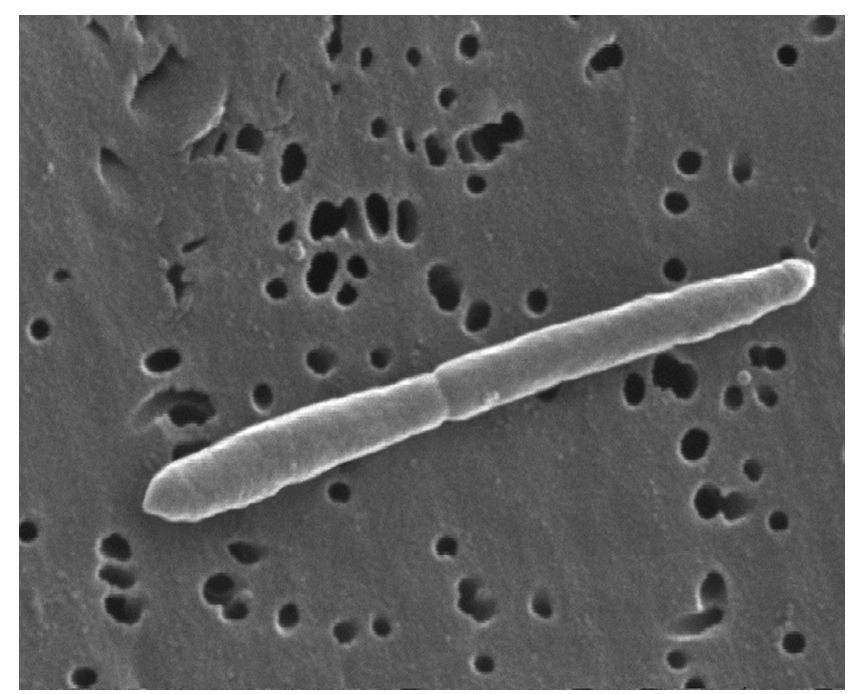

Figure 2. Scanning electron micrograph of $S$. termitidis NCTC $11300^{\top}$. (J. Carr, CDC, Atlanta, Georgia). More EM photos of the organism can be found at http://phil.cdc.gov/phi 
Table 1. Classification and general features of S. termitidis NCTC 11300 according to the MIGS recommendations [15]

\begin{tabular}{|c|c|c|c|}
\hline MIGS ID & Property & Term & Evidence code \\
\hline & \multirow{8}{*}{ Current classification } & Domain Bacteria & TAS [16] \\
\hline & & Phylum Fusobacteria & TAS [17] \\
\hline & & Class 'Fusobacteria' & TAS [17] \\
\hline & & Order 'Fusobacteriales' & TAS [17] \\
\hline & & Family 'Leptotrichiaceae' & TAS [18] \\
\hline & & Genus Sebaldella & TAS $[1,19]$ \\
\hline & & Species Sebaldella termitidis & TAS $[1,19]$ \\
\hline & & Type strain NCTC 11300 & TAS [1] \\
\hline & Gram stain & Gram negative & TAS [1] \\
\hline & Cell shape & $\begin{array}{l}\text { rod-shaped, with central swellings; } \\
\text { occur singly, in pairs and in filaments }\end{array}$ & TAS [1] \\
\hline & Motility & nonmotile & TAS [1] \\
\hline & Sporulation & nonsporulating & TAS [2] \\
\hline & Temperature range & mesophile & NAS \\
\hline & Optimum temperature & not determined & \\
\hline & Salinity & not reported & \\
\hline \multirow[t]{3}{*}{ MIGS-22 } & Oxygen requirement & obligate anaerobic & TAS [1] \\
\hline & Carbon source & glucose and other sugars & TAS [1] \\
\hline & Energy source & fermentation of glucose and other sugars & TAS [1] \\
\hline MIGS-6 & Habitat & bacterial flora of termite gastrointestinal tract & TAS [1] \\
\hline MIGS-15 & Biotic relationship & unknown & \\
\hline \multirow[t]{3}{*}{ MIGS-14 } & Pathogenicity & none reported & NAS \\
\hline & Biosafety level & 2 & TAS $[20]$ \\
\hline & Isolation & posterior intestinal content of termites & TAS [2] \\
\hline MIGS-4 & Geographic location & unknown & \\
\hline MIGS-5 & Sample collection time & 1962 or before & TAS $[1,2]$ \\
\hline MIGS-4.1 & Latitude & not reported & \\
\hline MIGS-4.2 & Longitude & not reported & \\
\hline MIGS-4.3 & Depth & not reported & \\
\hline MIGS-4.4 & Altitude & not reported & \\
\hline
\end{tabular}

Evidence codes - IDA: Inferred from Direct Assay (first time in publication); TAS: Traceable Author Statement (i.e., a direct report exists in the literature); NAS: Non-traceable Author Statement (i.e., not directly observed for the living, isolated sample, but based on a generally accepted property for the species, or anecdotal evidence). These evidence codes are from of the Gene Ontology project [21]. If the evidence code is IDA, then the property was directly observed for a live isolate by one of the authors or an expert mentioned in the acknowledgements.

\section{Chemotaxonomy}

The cell wall structure of strain NCTC $11300^{\mathrm{T}}$ has not yet been reported. Nonhydroxylated and 3hydroxyated fatty acids were present [1]. The major long chain fatty acids are saturated and monounsaturated straight chain acids: $\mathrm{C}_{16: 0}$ (37\%) and $\mathrm{C}_{18: 1}(41 \%)$, with methyl branched acids being absent [1], as opposed to straight-chain saturated, anteiso- and iso-methyl branched-chain acids in members of the genus Bacteroides, which are missing the monounsaturated acids [1]. Menaquinones were not detected, as opposed to members of the genus Bacteroides [1].

\section{Genome sequencing and annotation Genome project history}

This organism was selected for sequencing on the basis of its phylogenetic position, and is part of the Genomic Encyclopedia of Bacteria and Archaea project [22]. The genome project is deposited in the Genome OnLine Database [12] and the complete genome sequence is deposited in GenBank. Sequencing, finishing and annotation were performed by the DOE Joint Genome Institute (JGI). A summary of the project information is shown in Table 2. 
Table 2. Genome sequencing project information

\begin{tabular}{lll}
\hline MIGS ID & Property & Term \\
\hline MIGS-31 & Finishing quality & Finished \\
MIGS-28 & Libraries used & $\begin{array}{l}\text { One genomic 8kb pMCL200 library, one 454 } \\
\text { pyrosequence library and one Illumina library }\end{array}$ \\
MIGS-29 & Sequencing platforms & Sanger, 454 Titanium, Illumina \\
MIGS-31.2 & Sequencing coverage & $9.2 \times$ Sanger; 30.3× 454 Titanium \\
MIGS-30 & Assemblers & Newbler, phrap \\
MIGS-32 & Gene calling method & Prodigal, GenePRIMP \\
& INSDC ID & CP001739 (chromosome), \\
& Genbank Date of Release & CP001740, CP001741 (plasmids) \\
& GOLDember 19, 2009 \\
& NCBI project ID & Gc01144 \\
& Database: IMG-GEBA & 29539 \\
& Source material identifier & ATCC 33386 \\
& Project relevance & Tree of Life, GEBA \\
\hline
\end{tabular}

\section{Growth conditions and DNA isolation}

S. termitidis NCTC $11300^{\mathrm{T}}$, ATCC $33386^{\mathrm{TM}}$, was grown anaerobically in ATCC medium 1490 (Modified chopped meat medium) [23] at $37^{\circ} \mathrm{C}$. DNA was isolated from cell paste using a basic CTAB extraction and then quality controlled according to JGI guidelines.

\section{Genome sequencing and assembly}

The genome was sequenced using a combination of Sanger and 454 sequencing platforms. All general aspects of library construction and sequencing can be found at http://www.jgi.doe.gov/. 454 Pyrosequencing reads were assembled using the Newbler assembler version 1.1.02.15 (Roche). Large Newbler contigs were broken into 4,966 overlapping fragments of $1,000 \mathrm{bp}$ and entered into assembly as pseudo-reads. The sequences were assigned quality scores based on Newbler consensus q-scores with modifications to account for overlap redundancy and to adjust inflated qscores. A hybrid 454/Sanger assembly was made using the parallel phrap assembler (High Performance Software, LLC). Possible mis-assemblies were corrected with Dupfinisher [24] or transposon bombing of bridging clones (Epicentre Biotechnologies, Madison, WI). Gaps between contigs were closed by editing in Consed, custom primer walk or PCR amplification. A total of 796 Sanger finishing reads were produced to close gaps, to resolve repetitive regions, and to raise the quality of the finished sequence. Illumina reads were used to improve the final consensus quality using an inhouse developed tool (the Polisher, unpublished).
The error rate of the completed genome sequence is less than 1 in 100,000 . Together all sequence types provided $39.5 \times$ coverage of the genome. The final assembly contains 45,934 Sanger and 760,187 pyrosequence reads.

\section{Genome annotation}

Genes were identified using Prodigal [25] as part of the Oak Ridge National Laboratory genome annotation pipeline, followed by a round of manual curation using the JGI GenePRIMP pipeline [26]. The predicted CDSs were translated and used to search the National Center for Biotechnology Information (NCBI) nonredundant database, UniProt, TIGRFam, Pfam, PRIAM, KEGG, COG, and InterPro databases. Additional gene prediction analysis and manual functional annotation was performed within the Integrated Microbial Genomes Expert Review (IMG-ER) platform [27].

\section{Genome properties}

The genome consists of a 4,418,842 bp long chromosome, and two plasmids with $54,160 \mathrm{bp}$ and 13,648 bp length, respectively, with a $33.4 \%$ GC content (Table 3 and Figure 3). Of the 4,264 genes predicted, 4,210 were protein-coding genes, and 54 RNAs; 59 pseudogenes were identified. The majority of the protein-coding genes (60.4\%) were assigned with a putative function while those remaining were annotated as hypothetical proteins. The distribution of genes into COGs functional categories is presented in Table 4. 
Table 3. Genome Statistics

\begin{tabular}{lrr}
\hline Attribute & \multicolumn{1}{c}{ Value } & \% of Total \\
\hline Genome size (bp) & $4,486,650$ & $100.00 \%$ \\
DNA coding region (bp) & $3,918,335$ & $87.33 \%$ \\
DNA G+C content (bp) & $1,497,450$ & $33.38 \%$ \\
Number of replicons & 3 & \\
Extrachromosomal elements & 2 & \\
Total genes & 4,264 & $100.00 \%$ \\
RNA genes & 54 & $1.27 \%$ \\
rRNA operons & 4 & \\
Protein-coding genes & 4,210 & $98.73 \%$ \\
Pseudogenes & 59 & $1.38 \%$ \\
Genes with function prediction & 2,576 & $60.41 \%$ \\
Genes in paralog clusters & 1,253 & $29.39 \%$ \\
Genes assigned to COGs & 2,299 & $60.95 \%$ \\
Genes assigned Pfam domains & 2,787 & $65.36 \%$ \\
Genes with signal peptides & 801 & $18.79 \%$ \\
Genes with transmembrane helices & 901 & $21.13 \%$ \\
CRISPR repeats & 1 & \\
\hline
\end{tabular}

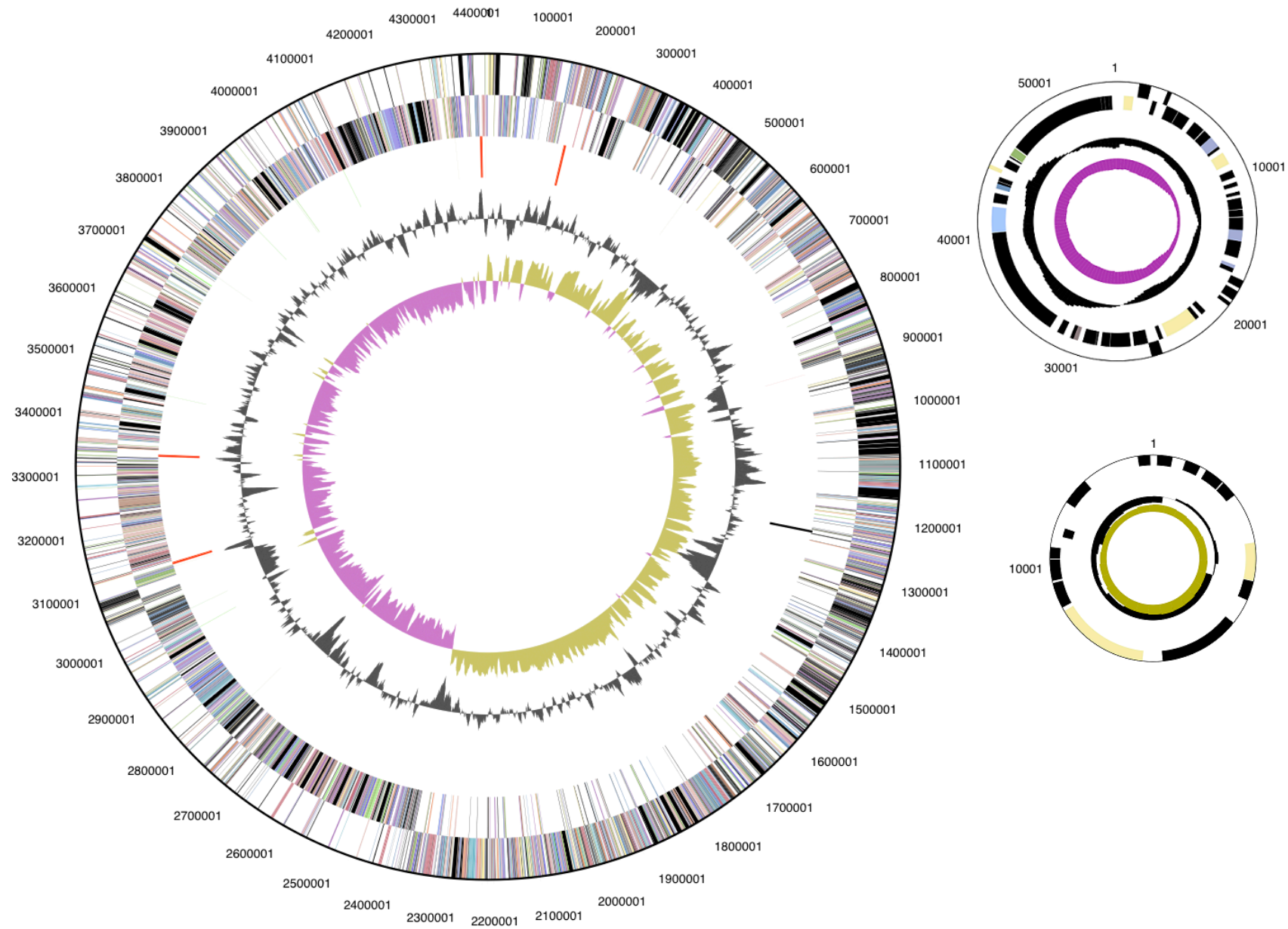

Figure 3. Graphical circular maps of the chromosome and the two plasmids. From outside to the center: Genes on forward strand (color by COG categories), Genes on reverse strand (color by COG categories), RNA genes (tRNAs green, rRNAs red, other RNAs black), GC content, GC skew. 
Table 4. Number of genes associated with the general COG functional categories

\begin{tabular}{crrl}
\hline Code & value & \%age & Description \\
\hline J & 152 & 3.6 & Translation, ribosomal structure and biogenesis \\
A & 0 & 0.0 & RNA processing and modification \\
K & 265 & 6.3 & Transcription \\
L & 130 & 3.1 & Replication, recombination and repair \\
B & 0 & 0.0 & Chromatin structure and dynamics \\
D & 22 & 0.5 & Cell cycle control, cell division, chromosome partitioning \\
Y & 0 & 0.0 & Nuclear structure \\
V & 47 & 1.1 & Defense mechanisms \\
T & 96 & 2.3 & Signal transduction mechanisms \\
M & 155 & 3.7 & Cell wall/membrane biogenesis \\
N & 17 & 0.4 & Cell motility \\
Z & 0 & 0.0 & Cytoskeleton \\
W & 0 & 0.0 & Extracellular structures \\
U & 41 & 1.0 & Intracellular trafficking, secretion and vesicular transport \\
O & 71 & 1.7 & Posttranslational modification, protein turnover, chaperones \\
C & 128 & 3.0 & Energy production and conversion \\
G & 468 & 11.1 & Carbohydrate transport and metabolism \\
E & 219 & 5.2 & Amino acid transport and metabolism \\
F & 93 & 2.2 & Nucleotide transport and metabolism \\
H & 106 & 2.5 & Coenzyme transport and metabolism \\
I & 59 & 1.4 & Lipid transport and metabolism \\
P & 105 & 2.5 & Inorganic ion transport and metabolism \\
Q & 32 & 0.8 & Secondary metabolites biosynthesis, transport and catabolism \\
R & 403 & 9.6 & General function prediction only \\
S & 241 & 5.7 & Function unknown \\
- & 1,665 & 39.5 & Not in COGs \\
\hline
\end{tabular}

\section{Acknowledgements}

We would like to gratefully acknowledge the help of Janice Carr (Centers of Disease Control, Atlanta, Georgia) for providing the EM photo of $S$. thermitidis NCTC $11300^{\mathrm{T}}$. This work was performed under the auspices of the US Department of Energy's Office of Science, Biological and Environmental Research Program, and by the University of California, Lawrence Berkeley Nation-

\section{References}

1. Collins MD, Shah HN. Reclassification of Bacteroides termitidis Sebald (Holdeman and Moore) in a new genus Sebaldella termitidis comb. nov. Int J Syst Bacteriol 1986; 36:349-350.

doi:10.1099/00207713-36-2-349

2. Sebald M. Etude sur les bacteries anaérobes gramnégatives asporulées. Thèse de I'Université Paris. Imprimerie Barnéoud S.A. Laval, France, 1962.

3. Holdeman LV, Kelly RW, Moore WEC. Genus Bacteroides, p. 604-631. In: Krieg NR, Holt JG (eds) Bergey's manual of systematic bacteriology, al Laboratory under contract No. DE-AC02-05CH11231, Lawrence Livermore National Laboratory under Contract No. DE-AC52-07NA27344, Los Alamos National Laboratory under contract No. DE-AC02-06NA25396, and Oak Ridge National Laboratory under contract DEAC05-000R22725

Vol. 1. The Williams \& Wilkins Co., Baltimore. 1984

4. Shah HN, Collins MD. Genus Bacteroides: a chemotaxonomical perspective. I Appl Bacteriol 1983; 55:403-416. PubMed

5. Paster BJ, Ludwig W, Weisburg WG, Stackebrandt E, Hespell RB, Hahn CM, Reichenbach H, Stetter $\mathrm{KO}$, Woese CR. A phylogenetic grouping of the Bacteroides, Cytophagas, and certain Flavobacteria. Syst Appl Microbiol 1985; 6:34-42. 
6. Rivière $D$, Desvignes $V$, Pelletier $E$, Chaussonnerie S, Guermazi S, Weissenbach J, Li T, Camacho $\mathrm{P}$, Sghir A. Towards the definition of a core of microorganisms involved in anaerobic digestion of sludge. ISME / 2009; 3:700-714. PubMed doi:10.1038/ismej.2009.2

7. Lehman RM, Lundgren JG, Petzke LM. Bacterial communities associated with the digestive tract of the predatory ground beetle, Poecilus chalcites, and their modifications by laboratory rearing ans antibiotic treatment. Microb Ecol 2009; 57:349358. $\underline{\text { PubMed doi:10.1007/s00248-008-9415-6 }}$

8. Chun J, Lee JH, Jung Y, Kim M, Kim S, Kim BK, Lim YW. EzTaxon: a web-based tool for the identification of prokaryotes based on $16 \mathrm{~S}$ ribosomal RNA gene sequences. Int / Syst Evol Microbiol 2007; 57:2259-2261. PubMed doi:10.1099/ijs.0.64915-0

9. Castresana J. Selection of conserved blocks from multiple alignments for their use in phylogenetic analysis. Mol Biol Evol 2000; 17:540-552. PubMed

10. Lee C, Grasso C, Sharlow MF. Multiple sequence alignment using partial order graphs. Bioinformatics 2002; 18:452-464. PubMed doi:10.1093/bioinformatics/18.3.452

11. Stamatakis A, Hoover $P$, Rougemont J. A rapid bootstrap algorithm for the RAxML web servers. Syst Biol 2008; 57:758-771. PubMed doi:10.1080/10635150802429642

12. Liolios K, Chen IM, Mavromatis K, Tavernarakis N, Hugenholtz P, Markowitz VM, Kyrpides NC. The Genomes On Line Database (GOLD) in 2009: status of genomic and metagenomic projects and their associated metadata. Nucleic Acids Res 2010; 38:D346-D354. PubMed doi:10.1093/nar/gkp848

13. Ivanova N, Gronow S, Lapidus A, Copeland A, Glavina Del Rio T, Nolan M, Lucas S, Cheng F, Tice H, Cheng JF, et al. Leptotrichia buccalis type strain (C-1013-b $\left.{ }^{\top}\right)$. Stand Genomic Sci 2009; 1:126-132. doi:10.4056/sigs.1854

14. Nolan M, Gronow S, Lapidus A, Ivanova N, Copeland A, Lucas S, Glavina Del Rio T, Chen F, Tice H, Pitluck S, et al. Streptobacillus moniliformis type strain $\left(9901^{\mathrm{T}}\right)$. Stand Genomic Sci 2009; 1:300-397. doi:10.4056/sigs.48727

15. Field D, Garrity G, Gray T, Morrison N, Selengut J, Sterk P, Tatusova T, Thomson N, Allen MJ, Angiuoli SV, et al. The minimum information about a genome sequence (MIGS) specification. Nat
Biotechnol 2008; 26:541-547. PubMed

doi:10.1038/nbt1360

16. Woese CR, Kandler O, Wheelis ML. Towards a natural system of organisms: proposal for the domains Archaea, Bacteria, and Eucarya. Proc Natl Acad Sci USA 1990; 87:4576-4579. PubMed doi:10.1073/pnas.87.12.4576

17. Garrity GM, Holt JG. Taxonomic Outline of the Archaea and Bacteria. In: Garrity GM, Boone DR, Castenholz RW (eds), Bergey's Manual of Systematic Bacteriology, Second Edition, Volume 1, Springer, New York, 2001, p. 155-166.

18. Ludwig W, Euzeby J, Whitman WG. Draft taxonomic outline of the Bacteroidetes, Planctomycetes, Chlamydiae, Spirochaetes, Fibrobacteres, Fusobacteria, Acidobacteria, Verrucomicrobia, Dictyoglomi, and Gemmatimonadetes. http://www.bergeys.org/outlines/Bergeys Vol 4 Outline.pdf. Taxonomic Outline 2008.

19. Skerman VBD, McGowan V, Sneath PHA. Approved Lists of Bacterial Names. Int / Syst Bacteriol 1980; 30:225-420. doi:10.1099/00207713$\underline{30-1-225}$

20. CDC's Office of Health and Safety. http://www.cdc.gov/od/ohs/biosfty/bmbl5/bmbl5t oc.htm

21. Ashburner M, Ball CA, Blake JA, Botstein D, Butler H, Cherry JM, Davis AP, Dolinski K, Dwight SS, Eppig JT, et al. Gene Ontology: tool for the unification of biology. Nat Genet 2000; 25:25-29. $\underline{\text { PubMed doi:10.1038/75556 }}$

22. Wu D, Hugenholtz P, Mavromatis K, Pukall R, Dalin E, Ivanova NN, Kunin V, Goodwin L, Wu $M$, Tindall BJ, et al. A phylogeny-driven genomic encyclopaedia of Bacteria and Archaea. Nature 2009; 462:1056-1060. PubMed doi:10.1038/nature08656

23. Growth media used at ATCC: http://www.atcc.org/Attachments/2718.pdf

24. Sims D, Brettin T, Detter J, Han C, Lapidus A, Copeland A, Glavina Del Rio T, Nolan M, Chen F, Lucas $\mathrm{S}$, et al. Complete genome sequence of $\mathrm{Ky}$ tococcus sedentarius type strain $\left(541^{\top}\right)$. Stand Genomic Sci 2009; 1:12-20. doi:10.4056/sigs.761

25. Hyatt D, Chen GL, Locascio PF, Land ML, Larimer FW, Hauser LJ. Prodigal: Prokaryotic Dynamic Programming Genefinding Algorithm. BMC Bioinformatics 2010; 11:119. PubMed doi:10.1186/1471-2105-11-119

26. Pati A, Ivanova N, Mikhailova N, Ovchinikova G, Hooper SD, Lykidis A, Kyrpides NC. GenePRIMP: 
A Gene Prediction Improvement Pipeline for microbial genomes. Nat Methods (In press).

27. Markowitz VM, Ivanova NN, Chen IMA, Chu K, Kyrpides NC. IMG ER: a system for microbial ge- nome annotation expert review and curation. Bioinformatics 2009; 25:2271-2278. PubMed

doi:10.1093/bioinformatics/btp393 